\title{
EXCESS OF LOSS REINSURANCE WITH REINSTATEMENTS REVISITED
}

\author{
BY \\ WERNER HÜRLIMANN
}

\begin{abstract}
The classical evaluation of pure premiums for excess of loss reinsurance with reinstatements requires the knowldege of the claim size distribution of the insurance risk. In the situation of incomplete information, where only a few characteristics of the aggregate claims to an excess of loss layer can be estimated, the method of stop-loss ordered bounds yields a simple analytical distribution-free approximation to pure premiums of excess of loss reinsurance with reinstatements. It is shown that the obtained approximation is enough accurate for practical purposes and improves the analytical approximations obtained using either a gamma, translated gamma, translated inverse Gaussian or a mixture of the last two distributions.
\end{abstract}

\section{KEYWORDS}

Excess of loss, reinstatement, aggregate deductible, aggregate limit, compound Poisson, Pareto, stop-loss order

\section{INTRODUCTION}

Despite their importance in practice, excess of loss reinsurance with reinstatements has been rather neglected in the actuarial literature. The main papers on the subject have been Simon(1972) and Sundt(1991). More recent discussions include Walhin(2001/2002), Walhin and Paris(2000/2001a/b), Mata(2000), Hess and Schmidt(2004).

The classical evaluation of pure premiums for excess of loss reinsurance with reinstatements, which is based on the collective model of risk theory, requires the knowledge of the claim size distribution and is exemplified in Sundt(1991). However, in practice, there is often incomplete information and only a few characteristics of the aggregate claims to an excess of loss layer can be estimated. This information includes the expected number of claims as well as the mean, variance and range of the claim size. In this situation, the method developed in Hürlimann(1996) (see also the applications in Hürlimann(2001/2003)) yields simple analytical distribution-free approximations to pure premiums of excess of loss 
reinsurance with reinstatements. As numerical examples suggest, the calculation requires usually only few operations and the obtained approximation is often quite accurate for practical purposes. Under certain conditions it improves the analytical approximations obtained from the recent proposals in Chaubey et al.(1998). The paper is organized as follows.

Sections 2 and 3 follow closely Sundt(1991). Section 2 recalls the main types of excess of loss reinsurance including aggregate deductibles, aggregate limits and reinstatements. The main formula by Sundt(1991) for the calculation of pure premiums for excess-of-loss reinsurance with equal reinstatements is presented in Section 3. The core of our analytical distribution-free approximation method is summarized in Section 4, which is based on Hürlimann(1996). There, we compare the numerical results by Sundt(1991) with those obtained using the distributionfree approach. The final Section 5 compares results obtained using the analytical approach by Sundt(1991) with those obtained from analytical approximations using a gamma, translated gamma, translated inverse Gaussian and a mixture of the last two distributions, as well as with those obtained using the distribution-free approach and the rate on line method introduced by Walhin(2001).

\section{TYPES OF EXCESS OF LOSS REINSURANCE}

The non-proportional reinsurance covers discussed in the present paper have been described in detail in Sundt(1991). Let us recall the main definitions and notations.

Given an insurance portfolio over a one-year period, let $N$ denote the number of claims occurring during the year and $Y_{i}$ the $i$-th claim size, $i=1, \ldots, N$.

An excess of loss reinsurance or for short an $X L$ reinsurance for the layer $m$ in excess of $\ell$, written $m \times s \quad \ell$, covers the part of each claim that exceeds the deductible $\ell$ but with a limit $m$ on the payment of each claim, that is the reinsurer covers for each claim

$$
Z_{i}=\min \left\{\left(Y_{i}-\ell\right)_{+}, m\right\}, \quad i=1, \ldots, N,
$$

where $(x)_{+}=x$ if $x>0$ and $(x)_{+}=0$ if $x \leq 0$. Let $X$ denote the aggregate claims to the layer, that is the random sum

$$
X=\sum_{i=1}^{N} Z_{i} .
$$

An $X L$ reinsurance for the layer $m$ xs $\ell$ with aggregate deductible $L$, written $m \times s \quad$ \& xs $L$, covers only the part of the aggregate claims to the layer that exceeds $L$, that is

$$
X_{L}=(X-L)_{+} .
$$

In case there is also an aggregate limit $M$ to the layer, the reinsurer covers the aggregate claims to the layer that exceeds $L$ but with a limited payment of $M$, that is 


$$
X_{L, M}=\min \left\{(X-L)_{+}, M\right\},
$$

and the cover is called an $X L$ reinsurance for the layer $m x s \ell$ with aggregate layer $M x s L$. If the aggregate limit $M$ is a whole multiple of the limit $m$, that is if $M=(\mathrm{K}+1) m$, one speaks of an XL reinsurance for the layer $m x s \quad l$ in the aggregate with $K$ reinstatements. The reinsurance coverage of this $\mathrm{XL}$ reinsurance is given by

$$
X_{L}^{K}=\min \left\{(X-L)_{+},(K+1) m\right\} .
$$

In this situation, the reinsurance has to be reinstated if the aggregate payment exceeds a whole multiple of the limit, that is $K \geq 1$. If $K=0$ there are no reinstatements. In practice, there are free and paid reinstatements. A reinstatement premium is expressed as percentage of the initial premium $P_{L}^{K}$, say $c_{k} P_{L}^{K}$ for the $k$-th reinstatement with $c_{k} \geq 0$, and it is paid pro rata of the claims to the layer. If $c_{k}=0$ the $k$-th reinstatement is free. The $k$-th reinstatement with premium $c_{k} P_{L}^{K}$ covers the amount

$$
r_{L}^{k}=\min \left\{(X-k m-L)_{+}, m\right\}, \quad k=1, \ldots, K .
$$

In this terminology the 0 -th reinstatement is set at the initial premium $P_{L}^{K}$ and covers the original layer, that is

$$
r_{L}^{0}=\min \left\{(X-L)_{+}, m\right\} .
$$

Since the reinstatement is paid pro rata, the random premium for the $k$-th reinstatement is $\frac{r_{L}^{k-1}}{m} c_{k} P_{L}^{K}$. The random total premium income required for this $\mathrm{XL}$ reinsurance equals

$$
{ }_{t o t} P_{L}^{K}=P_{L}^{K} \cdot\left(1+\frac{1}{m} \sum_{k=1}^{K} c_{k} r_{L}^{k-1}\right) .
$$

The aggregate claims paid by the reinsurer for this XL reinsurance clearly satisfies the identity

$$
\sum_{k=0}^{K} r_{L}^{k}=X_{L}^{K}
$$

with $X_{L}^{K}$ defined above in (2.5).

\section{Pure Premiums for XL Reinsurance With Reinstatements}

To calculate premiums for XL reinsurance with reinstatements, one needs to know the distribution function $F_{X}(x)=\operatorname{Pr}(X \leq x)$ of the aggregate claims $X$ to the layer $m x_{s} \ell$ defined in (2.2), as well as the associated stop-loss transform 
defined by $\pi_{X}(x)=E\left[(X-x)_{+}\right]=\int_{x}^{\infty} \bar{F}_{X}(x) d x$, where $\bar{F}_{X}(x)=1-F_{X}(x)$ denotes the survival probability function. Clearly, the pure premium should satisfy the expected value equation $E\left[{ }_{t o t} P_{L}^{K}\right]=E\left[X_{L}^{K}\right]$, that is by (2.8) the equation

$$
P_{L}^{K} \cdot\left(1+\frac{1}{m} \sum_{k=1}^{K} c_{k} E\left[r_{L}^{k-1}\right]\right)-E\left[X_{L}^{K}\right]
$$

which expresses the fact that the expected premium income should be equal to the expected claim payments.

For simplicity consider only the practical case of equal reinstatements $c_{k}=c, k=1, \ldots, K$ (e.g. $c=100 \%, 40 \%$ or $c=0$ if the reinstatements are free). Then (3.1) reads

$$
P_{L}^{K} \cdot\left(1+\frac{c}{m} E\left[X_{L}^{K-1}\right]\right)=E\left[X_{L}^{K}\right]
$$

But $X_{L}^{k}=\min \left\{(X-L)_{+},(k+1) m\right\}=(X-L)_{+}-(X-L-(k+1) m), k=1, \ldots, K$, and using the definition of the stop-loss transform, one obtains the main premium formula (e.g. Sundt(1991), formula following (3.4)):

$$
P_{L}^{K}=\frac{\pi_{X}(L)-\pi_{X}(L+(K+1) m)}{1+c \cdot \frac{\pi_{X}(L)-\pi_{X}(L+K m)}{m}} .
$$

In particular, if $c=0$ and $K \rightarrow \infty$ (infinitely many free reinstatements) and $L=0$ (no aggregate deductible) one has $P_{0}^{\infty}=E[X]$, which is the pure premium of the usual XL reinsurance with layer $m$ xs $\ell$.

\section{Distribution-FreE APPROXIMATIONS}

The formula (3.3) shows that the pure premium of the XL reinsurance with fixed reinstatements depends on the knowledge of the stop-loss transform associated to the aggregate claim

$$
X=\sum_{i=1}^{N} Z_{i}=\sum_{i=1}^{N} \min \left\{\left(Y_{i}-\ell\right)_{+}, m\right\}
$$

defined in (2.1) and (2.2).

In practice, the evaluation of the stop-loss transform of $X$ relies often on numerical methods, especially Panjer like recursive algorithms or Fast Fourier Transform methods. The calculations in Sundt(1991) are based on the well-known Panjer recursion, which requires the distribution of the claim size $Y_{i}$ in discrete form. The present distribution-free approach uses only a few characteristics of $X$, namely the expected number of claims $\lambda=E[N]$, the mean $\mu=E\left[Z_{i}\right]$ and the variance $\sigma^{2}=\operatorname{Var}\left[Z_{i}\right]$ of the $i$-th excess of loss claim size $Z_{i}$. Since the latter random variable is bounded, its range 
$[0, m]$ is also known. In this situation, the method developed in Hürlimann(1996) yields simple analytical lower and upper bounds to the stop-loss transform of $X$, which directly provide distribution-free approximations to pure premiums of the $\mathrm{XL}$ reinsurance with reinstatements. It is shown that the use of the upper bound only does not always produce conservative pure premiums. Therefore, a calculation using the lower bound as well as the average of these approximations might be an attractive practical alternative.

Recall the essential idea of the distribution-free approach. One assumes that the XL claim size $Z=Z_{i}$ belongs to the set $D=D([0, m] ; \mu, \sigma)$ of all random variables with fixed mean $\mu=E[Z]$, variance $\sigma^{2}=\operatorname{Var}[Z]$ and support contained in the interval $[0, m]$. Consider the quantities

$v=\left(\frac{\sigma}{\mu}\right)^{2} \quad:$ the relative variance of the claim size $Z$

$v_{0}=\frac{m-\mu}{\mu}:$ the maximal relative variance over the set $D$

$v_{r}=\frac{v}{v_{0}} \quad:$ the relative variance expressed in terms of the maximal relative variance

The main result of Section 2 in Hürlimann(1996) provides the construction of explicit stop-loss ordered random variables $Z_{*}, Z^{*}$ with the property $Z_{*}{ }_{s l} Z{ }_{s l} Z^{*}$ for all $Z \in D$, that is $\pi_{Z_{*}}(x) \leq \pi_{Z}(x) \leq \pi_{Z^{*}}(x)$ for all $x \geq 0$, and all $Z \in D$, where $\pi_{Z}(x)=E\left[(Z-x)_{+}\right]$denotes the stop-loss transform of the random variable $Z$. The distributions $F_{*}(z)$ and $F^{*}(z)$ of the normalized random variables $\left(Z_{*}-\mu\right) / \mu$ and $\left(Z^{*}-\mu\right) / \mu$ are summarized in Table 4.1.

TABLE 4.1

STOP-LOSS ORDERED EXTREMAL DISTRIBUTIONS

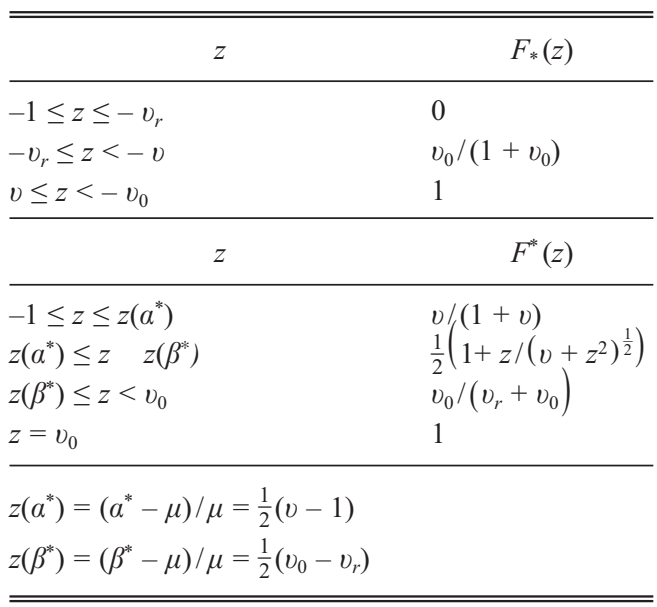


The idea of the distribution-free approach consists to replace the claim size $Z$ by the stop-loss ordered bounds $Z_{*}, Z^{*}$, and more precisely a stop-loss ordered discrete version $Z_{d}^{*}$ of $Z^{*}$. Then the aggregate claims to the layer $m x s \quad l$ is also stop-loss ordered such that

$$
X_{*}=\sum_{i=1}^{N} Z_{*_{i}} \leq_{s l} X=\sum_{i=1}^{N} Z_{i} \leq_{s l} X^{*}=\sum_{i=1}^{N} Z_{i}^{*} \leq X_{d}^{*}=\sum_{i=1}^{N} Z_{d, i}^{*} .
$$

Clearly, replacing $\pi_{X}(x)$ in (3.3) by $\pi_{X_{*}}(x)$ and $\pi_{X_{d}^{*}}(x)$ will provide lower and upper bounds to XL premiums with reinstatements. The present paper offers an analysis of the approximation obtained replacing $\pi_{X}(x)$ in (3.3) by $\pi_{X_{d}^{*}}(x)$ and $\pi_{X_{*}}(x)$.

From a statistical viewpoint this method has the advantage to be distributionfree, that is only dependent on the few risk characteristics $\lambda, \mu, \sigma$ and $m$. The numerical illustrations in Section 5 suggest that the distribution-free approximations $\pi_{X^{*}}(x)$ and $\pi_{X_{d}^{*}}(x)$ are very good approximations, especially for a small number of expected claims and higher layers. However, it is not always an upper bound to the pure premium. It is worth to investigate this matter a bit further. Denote the distribution-free approximation to the pure premium by

$$
P_{L}^{* K}=\frac{\pi_{X^{*}}(L)-\pi_{X^{*}}(L+(K+1) m)}{1+c \cdot \frac{\pi_{X^{*}}(L)-\pi_{X^{*}}(L+K m)}{m}} .
$$

In the particular case of free reinstatements $c=0$ and no aggregate deductible $L=0$, the approximate premium is always anticonservative provided the mean XL claim size remains fixed.

Proposition 4.1. For stop-loss ordered claim sizes with equal means, the approximate premium is always lower than the exact premium if the reinstatements are free and there is no aggregate deductible.

Proof. Noting that $\pi_{X}(0)=\pi_{X^{*}}(0)=E[X]$ and using the other assumptions, one sees that $P_{0}^{K}-P_{0}^{* K}=\pi_{X^{*}}((K+1) m)-\pi_{X}((K+1) m)$, which is always non-negative by (4.2).

A look at the case of free reinstatements but positive aggregate deductible shows that the approximate premium is conservative provided the following inequality holds:

$$
\pi_{X^{*}}(L)-\pi_{X}(L) \geq \pi_{X^{*}}(L+(K+1) m)-\pi_{X}(L+(K+1) m) .
$$

This is always fulfilled when the right-hand side becomes smaller and smaller. This is likely to be the case for a higher number of reinstatements, higher capacities and deductibles. As the numerical examples in Section 5 suggest, this is less likely fulfilled for small capacities and deductibles and higher number of expected claims. In any case, the approximation cannot be better than the smallest error of approximation $\pi_{X^{*}}(L)-\pi_{X}(L)$ for large $(K+1) m$, which will be maximum for the aggregate deductible satisfying the equation $F_{X^{*}}(L)=F_{X}(L)$. 
From a computational viewpoint the distribution-free approach is easy to implement. We assume that the aggregate claims $\sum_{i=1}^{N} Y_{i}$ of the original insurance portfolio is compound Poisson distributed such that $N$ is Poisson distributed with mean parameter $\lambda=E[N]$. Calculations for other claim number distributions will be similar but are not explicitly stated. As already stated, instead of $Z^{*}$ we will use a discrete stop-loss ordered approximation $Z_{d}^{*}$ of it such that $Z^{*}{ }_{s l} Z_{d}^{*}$. It is obtained by the method of mass dispersal, which is known to preserve the stop-loss order. From Hürlimann(1996), Section 3.2, one knows that $Z_{d}^{*}$ is a 4-atomic random variable with support $\left\{x_{0}, x_{1}, x_{2}, x_{3}\right\}$, and probabilities $\left\{p_{0}, p_{1}, p_{2}, p_{3}\right\}$ determined as follows:

$$
\begin{gathered}
x_{0}=0, \quad x_{1}=\frac{1}{2} \mu(1+v), \quad x_{2}=\mu\left(1+\frac{1}{2}\left(v_{0}-v_{r}\right)\right), \quad x_{3}=\mu\left(1+v_{0}\right), \\
p_{0}=\frac{v}{1+v}, \quad p_{1}=\frac{v_{0}-v}{\left(1+v_{0}\right)(1+v)}, \quad p_{2}=\frac{v_{0}-v}{\left(1+v_{0}\right)\left(v_{r}+v_{0}\right)}, \quad p_{3}=\frac{v_{r}}{v_{r}+v_{0}} .
\end{gathered}
$$

The stop-loss transform of $X_{d}^{*}$ can be evaluated using the series representation

$$
\pi_{X_{d}^{*}}(x)=\lambda \mu-x+e^{-\lambda\left(1-p_{0}\right)} \cdot \sum_{n_{1}, n_{2}, n_{3}=0}^{\infty} \frac{\left(\lambda p_{1}\right)^{n_{1}}\left(\lambda p_{2}\right)^{n_{2}}\left(\lambda p_{3}\right)^{n_{3}}}{n_{1} ! n_{2} ! n_{3} !}\left(x-\sum_{i=1}^{3} n_{i} x_{i}\right)_{+} .
$$

One notes that the infinite series are always finite sums because summation occurs only for $\sum_{i=1}^{3} n_{i} x_{i}<x$. In fact, often only very few terms must be evaluated. To evaluate the stop-loss transform obtained from the lower stop-loss bound $Z_{*}$ to $Z$, one notes that $Z_{*}$ is a biatomic random variable with support $\left\{x_{1}, x_{2}\right\}=\left\{\mu-\sigma^{2} /(m-\mu),(1+v) \mu\right\}$ and probabilities $\left\{p_{1}, p_{2}\right\}=\{1-\mu / m, \mu / m\}$. The required stop-loss transform is calculated using the formula

$$
\pi_{X_{*}}(x)=\lambda \mu-x+e^{-\lambda} \cdot \sum_{n_{1}, n_{2}}^{\infty} \frac{\left(\lambda p_{1}\right)^{n_{1}}\left(\lambda p_{2}\right)^{n_{2}}}{n_{1} ! n_{2} !}\left(x-\sum_{i=1}^{2} n_{i} x_{i}\right)_{+} .
$$

To illustrate the practical impact of the distribution-free approach, consider the example by Sundt(1991). Assume that the claim number $N$ is Poisson distributed with parameter $\lambda$ and the claim sizes $Y_{i}$ are Pareto distributed with scale parameter $O P$ (the so-called observation point) and index $\alpha$, that is

$$
\begin{gathered}
P(N=n)=\frac{\lambda^{n}}{n !} e^{-\lambda}, \quad n=0,1,2, \ldots, \\
P(Y \leq y)=1-\left(\frac{y}{O P}\right)^{-a}, \quad y \geq O P>0, a>0 .
\end{gathered}
$$

To implement the distribution-free method, one needs the first two moments of the claim size $Z=(Y-\ell)_{+}-(Y-m-\ell)_{+}$from an XL reinsurance with layer $m \times s \quad \ell$. One obtains through induction the recursive formula 
$E\left[Z^{n}\right]=O P^{n} \cdot\left(\frac{n}{n-\alpha}\right)\left\{\left(\frac{m+\ell}{O P}\right)^{n-a}-\left(\frac{\ell}{O P}\right)^{n-a}\right\}-\sum_{k=1}^{n-1}\left(\begin{array}{l}n \\ k\end{array}\right) \ell^{n-k} \cdot E\left[Z^{k}\right], \quad n=1,2, \ldots$

The parameters required to evaluate the approximate stop-loss transforms (4.7) and (4.8) are besides $\lambda$ and $m$, the mean $\mu=E[Z]$ and the variance $\sigma^{2}=\operatorname{Var}[Z]$ obtained from (4.11). The original analytical approach by Sundt(1991) requires an appropriate discretization of the claim size distribution, which is used to evaluate the stop-loss transform using Panjer's recursion. Based on the Pareto claim size (4.10), the distribution of the XL claim size reads

$$
F(z)=\left\{\begin{array}{l}
1-\left(\frac{\ell+z}{m}\right)^{-a}, 0 \leq z \leq m, \\
1, \quad z \geq m .
\end{array}\right.
$$

Applying the method of mass dispersal, one approximates $F(z)$ by a distribution on the $t+1$ points $i h, i=0, \ldots, t$, with $h=m / t$. Let $\bar{F}(z)=1-F(z)$ denote the survival function and let $f_{i}$ be the probability associated to the point $i h, i=0, \ldots, t$. It is not difficult to obtain the following formulas

$$
\begin{gathered}
f_{0}=\varepsilon_{1}-x_{1}+1-\left(\frac{\ell}{O P}\right)^{-a}, \\
f_{i}=x_{i}+\varepsilon_{i+1},-x_{i+1}, i=1, \ldots, t-1, \quad f_{t}=x_{t},
\end{gathered}
$$

where one sets

$$
\begin{gathered}
\varepsilon_{i}=\left(\frac{\ell+(i-1) h}{O P}\right)^{-a}-\left(\frac{\ell+i h}{O P}\right)^{-a}, \quad i=1, \ldots, t-1, \\
\varepsilon_{t}=\left(\frac{\ell+(t-1) h}{O P}\right)^{-a}, \quad x_{i}=\frac{\omega_{i}}{h}-(i-1) \cdot \varepsilon_{i}, \quad i=1, \ldots, t, \\
\omega_{i}=\frac{O P}{a-1} \cdot\left\{\left(\frac{\ell+(i-1) h}{O P}\right)^{-a+1}-\left(\frac{\ell+i h}{O P}\right)^{-a+1}\right\} \\
+(i-1) h\left(\frac{\ell+(i-1) h}{O P}\right)^{-a}-i h\left(\frac{\ell+i h}{O P}\right)^{-a}, i=1, \ldots, t-1, \\
\omega_{t}=\mu-\sum_{i=1}^{t-1} \omega_{i} .
\end{gathered}
$$

Sundt(1991) calculates pure premiums for $m=\ell=100, O P=100, \alpha=1.2, \lambda=0.5$. Table 4.2 compares the results of this analytical approach with those obtained using the distribution-free approach based on (4.7). With much less computational effort, in fact at most 11 terms are required in the formula (4.5), the approximate pure premiums are surprisingly accurate and in most cases on the safe side. Note that the case $K=5$ is compared with the case of infinitely many reinstatements in Sundt(1991). A more detailed study including the approximation by (4.8) is pursued in the next Section. 
TABLE 4.2

INITIAL PURE PREMIUMS SUNDT(1991) VS. DISTRIBUTION-FREE APPROACH (DF)

\begin{tabular}{cccccccc}
\hline \hline & & \multicolumn{2}{c}{$L=0$} & \multicolumn{2}{c}{$L=100$} & \multicolumn{2}{c}{$L=200$} \\
$\mathrm{~K}$ & $\mathrm{c}$ & Sundt & DF & Sundt & DF & Sundt & DF \\
\hline 0 & 0 & 27.85 & 27.73 & 4.088 & 4.176 & 0.3963 & 0.4238 \\
1 & 0 & 31.94 & 31.90 & 4.485 & 4.600 & 0.4247 & 0.4559 \\
& 1 & 24.98 & 24.98 & 4.309 & 4.416 & 0.4230 & 0.4540 \\
2 & 0 & 32.33 & 32.33 & 4.514 & 4.632 & 0.4264 & 0.4579 \\
& 1 & 24.51 & 24.51 & 4.319 & 4.428 & 0.4245 & 0.4558 \\
$5(\infty)$ & 0 & 32.36 & 32.36 & 4.515 & 4.634 & 0.4263 & 0.4580 \\
& 1 & 24.45 & 24.45 & 4.320 & 4.429 & 0.4246 & 0.4559 \\
\hline \hline
\end{tabular}

\section{AnALYTiCAl APPROXIMATIONS}

In view of the very accurate approximations obtained with the distribution-free approach in Table 4.1, it is natural to ask whether other analytical approximations of the aggregate claims to the layer $m$ xs $\ell$ perform similarly. From Chaubey et al.(1998) one knows that the approximations based on the gamma, translated gamma, translated inverse Gaussian and a mixture of the last two distributions yield usually good approximations of aggregate claims distributions. Like the distribution-free approach, these approximations are based on the method of moments an do not use Panjer's recursive formula as in the original analytical approach by Sundt(1991). Even more, Chaubey et al.(1998) claim that the latter mixture seems to be a superb approximation to the aggregate claims distribution. The compound Poisson approximations use at most 5 parameters, namely the expected number of claims $\lambda=E[N]$ and the first four moments $E\left[Z^{i}\right], i=1, \ldots, 4$, of the claim size. Recall the formulas required to evaluate the analytical approximations to the aggregate claims $X$.

\section{Gamma approximation}

The distribution of $X$ is approximated by a gamma distribution $\Gamma(\beta, a)$ with parameters $a=k_{X}^{-2}, \beta=\left(k_{X}^{2} \mu_{X}\right)^{-1}$ where $\mu_{X}, k_{X}$ are the mean and coefficient of variation of $X$ given by

$$
\mu_{X}=\lambda E[Z], \quad k_{X}^{2}=\frac{1}{\lambda} \cdot \frac{E\left[Z^{2}\right]}{E[Z]^{2}} .
$$

This approximation matches the mean and variance of $X$.

\section{Translated Gamma approximation}

According to Dickson and Waters(1993), it is natural to approximate $X$ by $X_{T G}=X_{G}+\gamma$, where $X_{G} \sim \Gamma(\beta, \alpha)$, with the parameters defined by 


$$
\alpha=4 \lambda \cdot \frac{E\left[Z^{2}\right]^{3}}{E\left[Z^{3}\right]^{2}}, \quad \beta=2 \cdot \frac{E\left[Z^{2}\right]}{E\left[Z^{3}\right]}, \quad \gamma=\lambda \cdot\left(E[Z]-2 \cdot \frac{E\left[Z^{2}\right]^{2}}{E\left[Z^{3}\right]}\right) .
$$

This approximation matches the mean, variance and skewness of $X$.

\section{Translated inverse Gaussian approximation}

Chaubey et al.(1998) propose to approximate $X$ by $X_{T I G}=X_{I G}+\delta$, where $X_{I G} \sim I G(a, b)$ has an inverse Gaussian distribution, with the parameters defined by

$$
a=3 \lambda \cdot \frac{E\left[Z^{2}\right]^{2}}{E\left[Z^{3}\right]}, \quad b=\frac{1}{3} \frac{E\left[Z^{3}\right]}{E\left[Z^{2}\right]}, \quad \delta=\lambda E[Z]-a
$$

This approximation matches the mean, variance and skewness of $X$.

\section{Mixture of translated gamma and translated inverse Gaussian}

Let $F_{T G}(x)=F_{G}(x-\gamma)$ and $F_{T I G}(x)=F_{I G}(x-\delta)$ be the distribution functions of the translated gamma and translated inverse Gaussian approximations. Following Chaubey(1989), the mixture defined by

$$
F_{\text {mix }}(x)=w \cdot F_{T G}(x)+(1-w) \cdot F_{T I G}(x)
$$

matches the mean, variance, skewness and kurtosis of $X$ provided

$$
w=\frac{\kappa_{X}-\kappa_{T I G}}{\kappa_{T G}-\kappa_{T I G}}
$$

where the kurtosis parameters are defined by

$$
\kappa_{X}=\frac{1}{\gamma} \cdot \frac{E\left[Z^{4}\right]}{E\left[Z^{2}\right]^{2}}, \quad \kappa_{T G}=\frac{6}{a}, \quad \kappa_{T I G}=15 \cdot \frac{b}{a} .
$$

Another very simple approximation to pure premiums for XL reinsurance with reinstatements is the rate on line method introduced by Walhin(2001). Consider the rate on line of a layer $m x s \ell$ defined by $R O L=\lambda \cdot E[Z] / m$, which is the premium of an unlimited free reinstatements treaty divided by the capacity. The rate on line method assumes that there are only total losses hitting the layer completely with the frequency $\theta=R O L$. If one assumes that $Z=m$ with probability one, and $N$ has the mean $\theta$, then the rate on line approximate premium is given by the formula

$$
P_{L}^{K}=m \cdot \frac{\pi_{N}\left(\frac{L}{m}\right)-\pi_{N}\left(\frac{L}{m}+(K+1)\right)}{1+c \cdot \frac{\pi_{N}\left(\frac{L}{m}\right)-\pi_{N}\left(\frac{L}{m}+K\right)}{m}} .
$$


In case $N$ is Poisson distributed with survival function $S(i ; \theta)=P(N>i)=1-$ $\sum_{j=0}^{i} e^{-\theta} \cdot \frac{\theta^{j}}{j !}$, and the aggregate deductible is an integer multiple of the cover $m$, one obtains the formulas

$$
\begin{gathered}
P_{L}^{K}=m \cdot S\left(\frac{L}{m} ; \theta\right), \quad \text { if } K=0, \\
P_{L}^{K}=m \cdot \frac{\sum_{i=\frac{L}{m}}^{\frac{L}{m}+K} S(i ; \theta)}{1+c \cdot \sum_{i=\frac{L}{m}}^{\frac{L}{m}+K-1} S(i ; \theta)}, \quad \text { if } K \geq 1 .
\end{gathered}
$$

The Tables 5.2 to 5.16 offer a sensitivity analysis of the different approximations by comparing the results obtained with the analytical approach by Sundt(1991) with those obtained using the above analytical approximations, the distribution-free approach and the rate on line method. It is important to note that the distribution-free approach always yields a lower and an upper bound obtained from (4.7) and (4.8). It is interesting to compare the average of these bounds with the results obtained from the other methods. The claim size has a Pareto distribution with observation point $O P=100$ and index $a$, and the claim number is Poisson distributed with parameter $\lambda$. We restrict the analysis to the more common case of free reinstatements $c=0$. The example by Sundt(1991) for $L=0,100,200$ and additional higher layers is studied in the Tables 5.2, 5.7 and 5.12. The other Tables consider similar examples with varying expected number of claims $\lambda=1,2,5,10$ and fixed Pareto index $\alpha=2.5$. Table 5.1 provides an overview of the three best approximations to the analytical premiums by Sundt(1991). In the situation of no aggregate deductible $L=0$, the distribution-free approach based on (4.7) is always best for the smaller expected number of claims up to $\lambda=2$, followed by the rate on line method and the Gamma approximation. In these situations, the mixture approximation by Chaubey et al.(1998) does not perform well. However, for the higher expected number of claims $\lambda=5,10$ and the lowest layer, the mixture approximation is best, followed by the translated gamma and the translated inverse Gaussian distributions. For $\lambda=5,10$, and the middle and higher layer, the distribution-free approach is again best, followed by the rate on line method and the Gamma approximation. By increasing the aggregate deductible, as for instance $L=100$, similar remarks can be made with the following differences. The distribution-free approach and the rate on line method are only best for even smaller expected number of claims and higher layers, and the translated inverse Gaussian takes the role of the Gamma as third best approximation in these cases. The mixture approximation is best for the lowest layer already when $\lambda \geq 2$. 
TABLE 5.1

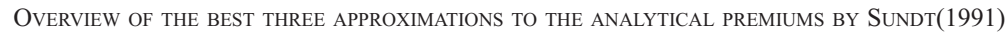

\begin{tabular}{|c|c|c|c|c|c|c|c|}
\hline \multirow[b]{2}{*}{ Table } & \multirow[b]{2}{*}{$\lambda$} & \multirow[b]{2}{*}{$a$} & \multirow[b]{2}{*}{$L$} & \multirow[b]{2}{*}{ Layer } & \multicolumn{3}{|c|}{ Choice } \\
\hline & & & & & 1 & 2 & 3 \\
\hline \multirow[t]{3}{*}{5.2} & 0.5 & 1.2 & 0 & $100-200$ & & & \\
\hline & & & & $200-300$ & DF & ROL & Gamma \\
\hline & & & & $300-400$ & & & \\
\hline \multirow[t]{3}{*}{5.3} & 1 & 2.5 & 0 & $100-200$ & & & \\
\hline & & & & $200-300$ & DF & ROL & Gamma \\
\hline & & & & $300-400$ & & & \\
\hline \multirow[t]{3}{*}{5.4} & 2 & 2.5 & 0 & $100-200$ & & & \\
\hline & & & & $200-300$ & DF & ROL & Gamma \\
\hline & & & & $300-400$ & & & \\
\hline \multirow[t]{3}{*}{5.5} & 5 & 2.5 & 0 & $100-200$ & Mixture & TG & TIG \\
\hline & & & & $200-300$ & $\mathrm{DF}$ & ROL & Gamma \\
\hline & & & & $300-400$ & $\mathrm{DF}$ & ROL & Gamma \\
\hline \multirow[t]{3}{*}{5.6} & 10 & 2.5 & 0 & $100-200$ & Mixture & $\mathrm{TG}$ & TIG \\
\hline & & & & $200-300$ & $\mathrm{DF}$ & ROL & Gamma \\
\hline & & & & $300-400$ & DF & ROL & Gamma \\
\hline \multirow[t]{3}{*}{5.7} & 0.5 & 1.2 & 100 & $100-200$ & & & \\
\hline & & & & $200-300$ & DF & ROL & TIG \\
\hline & & & & $300-400$ & & & \\
\hline \multirow[t]{3}{*}{5.8} & 1 & 2.5 & 100 & $100-200$ & TG & TIG & Mixture \\
\hline & & & & $200-300$ & DF & ROL & TIG \\
\hline & & & & $300-400$ & $\mathrm{DF}$ & ROL & TIG \\
\hline \multirow[t]{3}{*}{5.9} & 2 & 2.5 & 100 & $100-200$ & Mixture & $\mathrm{DF}$ & ROL \\
\hline & & & & $200-300$ & $\mathrm{DF}$ & ROL & TIG \\
\hline & & & & $300-400$ & $\mathrm{DF}$ & ROL & TIG \\
\hline \multirow[t]{3}{*}{5.10} & 5 & 2.5 & 100 & $100-200$ & Mixture & TG & TIG \\
\hline & & & & $200-300$ & TIG & TG & $\mathrm{DF}$ \\
\hline & & & & $300-400$ & $\mathrm{DF}$ & ROL & TIG \\
\hline \multirow[t]{3}{*}{5.11} & 10 & 2.5 & 100 & $100-200$ & Mixture & TG & TIG \\
\hline & & & & $200-300$ & TIG & TG & $\mathrm{DF}$ \\
\hline & & & & $300-400$ & $\mathrm{DF}$ & ROL & TIG \\
\hline
\end{tabular}


TABLE 5.2

XL PURE PREMIUMS WITH REINSTATEMENTS UNDER VARIOUS DISTRIBUTION APPROXIMATIONS, $\lambda=0.5, a=1.2, L=0, c=0$

\begin{tabular}{|c|c|c|c|c|c|c|c|c|c|}
\hline Deductible & 100 & & & & & & & & \\
\hline Limit & 200 & & & & & & & & \\
\hline \multirow[t]{6}{*}{ K } & G & TG & TIG & Mixture & DF up & DF down & DF av & ROL & Sundt \\
\hline & 26.29 & 31.42 & 31.85 & 30.19 & 27.72820 & 28.16825 & 27.94823 & 27.64775 & 27.84761 \\
\hline & 30.86 & 35.93 & 36.20 & 35.17 & 31.90438 & 32.03634 & 31.97036 & 31.88060 & 31.93604 \\
\hline & 31.96 & 36.52 & 36.80 & 35.73 & 32.32816 & 32.34222 & 32.33519 & 32.32464 & 32.33235 \\
\hline & 32.25 & 36.59 & 36.89 & 35.76 & 32.36032 & 32.36144 & 32.36088 & 32.35997 & 32.36069 \\
\hline & 32.35 & 36.60 & 36.91 & 35.76 & 32.36235 & 32.36236 & 32.36236 & 32.36235 & 32.36236 \\
\hline Deductible & 200 & & & & & & & & \\
\hline Limit & 300 & & & & & & & & \\
\hline \multirow[t]{6}{*}{ K } & G & TG & TIG & Mixture & DF up & DF down & DF av & ROL & Sundt \\
\hline & 14.13 & 19.32 & 19.73 & 18.13 & 15.60142 & 15.78502 & 15.69322 & 15.59322 & 15.61642 \\
\hline & 16.25 & 21.13 & 21.46 & 20.20 & 16.87891 & 16.89422 & 16.88657 & 16.87760 & 16.88120 \\
\hline & 16.76 & 21.33 & 21.67 & 20.35 & 16.94925 & 16.95008 & 16.94967 & 16.94914 & 16.94942 \\
\hline & 16.90 & 21.35 & 21.70 & 20.35 & 16.95215 & 16.95219 & 16.95217 & 16.95215 & 16.95216 \\
\hline & 16.95 & 21.36 & 21.70 & 20.35 & 16.95225 & 16.95225 & 16.95225 & 16.95225 & 16.95225 \\
\hline Deductible & 300 & & & & & & & & \\
\hline Limit & 400 & & & & & & & & \\
\hline \multirow[t]{6}{*}{ K } & G & TG & TIG & Mixture & DF up & DF down & $\mathrm{DF}$ av & ROL & Sundt \\
\hline & 9.43 & 14.03 & 14.46 & 12.79 & 10.61607 & 10.67877 & 10.64742 & 10.61419 & 10.61969 \\
\hline & 10.78 & 15.09 & 15.47 & 14.00 & 11.19875 & 11.20218 & 11.20047 & 11.19855 & 11.19913 \\
\hline & 11.10 & 15.20 & 15.58 & 14.07 & 11.22021 & 11.22034 & 11.22028 & 11.22020 & 11.22023 \\
\hline & 11.19 & 15.21 & 15.60 & 14.07 & 11.22081 & 11.22081 & 11.22081 & 11.22081 & 11.22081 \\
\hline & 11.22 & 15.21 & 15.60 & 14.07 & 11.22082 & 11.22082 & 11.22082 & 11.22082 & 11.22082 \\
\hline
\end{tabular}


TABLE 5.3

XL PURE PREMIUMS WITH REINSTATEMENTS UNDER VARIOUS DISTRIBUTION APPROXIMATIONS, $\lambda=0.5, \alpha=1.2, L=0, c=0$

\begin{tabular}{|c|c|c|c|c|c|c|c|c|c|}
\hline Deductible & 100 & & & & & & & & \\
\hline Limit & 200 & & & & & & & & \\
\hline K & G & TG & TIG & Mixture & DF up & DF down & DF av & ROL & Sundt \\
\hline 0 & 35.42 & 38.93 & 39.38 & 37.78 & 35.571 & 37.815 & 36.693 & 35.012 & 36.225 \\
\hline 1 & 41.47 & 45.01 & 45.28 & 44.32 & 42.211 & 42.735 & 42.473 & 42.016 & 42.395 \\
\hline 2 & 42.73 & 45.78 & 46.06 & 45.07 & 43.019 & 43.083 & 43.051 & 42.985 & 43.045 \\
\hline 3 & 43.01 & 45.87 & 46.17 & 45.12 & 43.091 & 43.096 & 43.094 & 43.087 & 43.094 \\
\hline 5 & 43.09 & 45.88 & 46.18 & 45.12 & 43.096 & 43.096 & 43.096 & 43.096 & 43.096 \\
\hline Deductible & 200 & & & & & & & & \\
\hline Limit & 300 & & & & & & & & \\
\hline \multirow[t]{6}{*}{ K } & G & TG & TIG & Mixture & DF up & DF down & DF av & ROL & Sundt \\
\hline & 9.31 & 13.17 & 13.59 & 11.99 & 10.19822 & 10.35706 & 10.27764 & 10.18356 & 10.22513 \\
\hline & 10.44 & 14.00 & 14.39 & 12.92 & 10.72204 & 10.72996 & 10.72600 & 10.72065 & 10.72432 \\
\hline & 10.67 & 14.07 & 14.47 & 12.96 & 10.73977 & 10.74003 & 10.73990 & 10.73970 & 10.73986 \\
\hline & 10.72 & 14.08 & 14.48 & 12.96 & 10.74021 & 10.74022 & 10.74022 & 10.74021 & 10.74021 \\
\hline & 10.74 & 14.08 & 14.48 & 12.95 & 10.74022 & 10.74022 & 10.74022 & 10.74022 & 10.74022 \\
\hline Deductible & 300 & & & & & & & & \\
\hline Limit & 400 & & & & & & & & \\
\hline \multirow[t]{6}{*}{ K } & G & TG & TIG & Mixture & DF up & DF down & $\mathrm{DF}$ av & ROL & Sundt \\
\hline & 3.91 & 6.47 & 6.92 & 5.17 & 4.39845 & 4.42014 & 4.40930 & 4.39707 & 4.40111 \\
\hline & 4.37 & 6.77 & 7.21 & 5.50 & 4.49525 & 4.49571 & 4.49548 & 4.49519 & 4.49535 \\
\hline & 4.46 & 6.79 & 7.24 & 5.51 & 4.49666 & 4.49666 & 4.49666 & 4.49666 & 4.49666 \\
\hline & 4.49 & 6.80 & 7.25 & 5.51 & 4.49667 & 4.49667 & 4.49667 & 4.49667 & 4.49667 \\
\hline & 4.50 & 6.80 & 7.25 & 5.51 & 4.49667 & 4.49667 & 4.49667 & 4.49667 & 4.49667 \\
\hline
\end{tabular}


TABLE 5.4

XL PURE PREMIUMS WITH REINSTATEMENTS UNDER VARIOUS DISTRIBUTION APPROXIMATIONS, $\lambda=2, \alpha=2.5, L=0, c=0$

\begin{tabular}{|c|c|c|c|c|c|c|c|c|c|}
\hline Deductible & 100 & & & & & & & & \\
\hline Limit & 200 & & & & & & & & \\
\hline \multirow[t]{6}{*}{ K } & G & TG & TIG & Mixture & DF up & DF down & DF av & ROL & Sundt \\
\hline & 61.67 & 62.74 & 63.16 & 61.65 & 59.10354 & 63.79292 & 61.44823 & 57.76533 & 60.66046 \\
\hline & 79.62 & 82.69 & 82.94 & 82.03 & 80.08163 & 82.68215 & 81.38189 & 79.12739 & 81.00793 \\
\hline & 84.47 & 87.02 & 87.20 & 86.54 & 85.14181 & 85.84695 & 85.49438 & 84.80093 & 85.41406 \\
\hline & 85.75 & 87.78 & 87.98 & 87.27 & 86.04843 & 86.16873 & 86.10858 & 85.96701 & 86.10128 \\
\hline & 86.16 & 87.92 & 88.14 & 87.35 & 86.19132 & 86.19283 & 86.19208 & 86.18918 & 86.19217 \\
\hline Deductible & 200 & & & & & & & & \\
\hline Limit & 300 & & & & & & & & \\
\hline \multirow[t]{6}{*}{ K } & G & TG & TIG & Mixture & DF up & DF down & $\mathrm{DF}$ av & ROL & Sundt \\
\hline & 18.12 & 22.91 & 23.32 & 21.78 & 19.38214 & 19.95334 & 19.66774 & 19.33008 & 19.47726 \\
\hline & 20.72 & 25.19 & 25.50 & 24.33 & 21.34185 & 21.39885 & 21.37035 & 21.33190 & 21.35799 \\
\hline & 21.29 & 25.43 & 25.75 & 24.52 & 21.47359 & 21.47743 & 21.47551 & 21.47263 & 21.47495 \\
\hline & 21.43 & 25.45 & 25.79 & 24.53 & 21.48017 & 21.48037 & 21.48027 & 21.48011 & 21.48025 \\
\hline & 21.48 & 25.46 & 25.79 & 24.52 & 21.48044 & 21.48044 & 21.48044 & 21.48044 & 21.48044 \\
\hline Deductible & 300 & & & & & & & & \\
\hline Limit & 400 & & & & & & & & \\
\hline \multirow[t]{6}{*}{ K } & G & $\mathrm{TG}$ & TIG & Mixture & DF up & DF down & DF av & ROL & Sundt \\
\hline & 7.73 & 11.55 & 11.99 & 10.31 & 8.60606 & 8.68901 & 8.64754 & 8.60080 & 8.61618 \\
\hline & 8.71 & 12.27 & 12.67 & 11.12 & 8.98219 & 8.98572 & 8.98396 & 8.98175 & 8.98296 \\
\hline & 8.92 & 12.33 & 12.74 & 11.15 & 8.99311 & 8.99321 & 8.99316 & 8.99309 & 8.99313 \\
\hline & 8.97 & 12.33 & 12.75 & 11.15 & 8.99334 & 8.99334 & 8.99334 & 8.99334 & 8.99334 \\
\hline & 8.99 & 12.34 & 12.75 & 11.15 & 8.99335 & 8.99335 & 8.99335 & 8.99335 & 8.99335 \\
\hline
\end{tabular}


TABLE 5.5

XL PURE PREMIUMS WITH REINSTATEMENTS UNDER VARIOUS DISTRIBUTION APPROXIMATIONS, $\lambda=5, \alpha=2.5, L=0, c=0$

\begin{tabular}{|c|c|c|c|c|c|c|c|c|c|}
\hline Deductible & 100 & & & & & & & & \\
\hline Limit & 200 & & & & & & & & \\
\hline K & G & TG & TIG & Mixture & DF up & DF down & $\mathrm{DF}$ av & ROL & Sundt \\
\hline 0 & 94.92 & 92.28 & 92.28 & 92.27 & 90.20 & 94.36 & 92.28 & 88.41 & 92.07 \\
\hline 1 & 160.26 & 159.23 & 159.43 & 158.72 & 155.21 & 163.48 & 159.34 & 151.84 & 158.50 \\
\hline 2 & 193.34 & 194.85 & 195.03 & 194.41 & 191.51 & 198.79 & 195.15 & 188.35 & 194.30 \\
\hline 3 & 207.36 & 209.32 & 209.39 & 209.14 & 207.51 & 211.40 & 209.45 & 205.53 & 209.04 \\
\hline 5 & 214.56 & 215.40 & 215.44 & 215.28 & 214.94 & 215.36 & 215.15 & 214.59 & 215.13 \\
\hline Deductible & 200 & & & & & & & & \\
\hline Limit & 300 & & & & & & & & \\
\hline K & G & TG & TIG & Mixture & DF up & DF down & $\mathrm{DF}$ av & ROL & Sundt \\
\hline 0 & 41.32 & 45.32 & 45.79 & 44.01 & 41.779 & 44.340 & 43.059 & 41.551 & 42.188 \\
\hline 1 & 50.44 & 55.25 & 55.50 & 54.55 & 51.823 & 52.471 & 52.147 & 51.714 & 51.999 \\
\hline 2 & 52.81 & 56.94 & 57.18 & 56.29 & 53.475 & 53.584 & 53.530 & 53.449 & 53.513 \\
\hline 3 & 53.45 & 57.20 & 57.46 & 56.49 & 53.679 & 53.693 & 53.686 & 53.675 & 53.685 \\
\hline 5 & 53.68 & 57.24 & 57.51 & 56.50 & 53.701 & 53.701 & 53.701 & 53.701 & 53.701 \\
\hline Deductible & 300 & & & & & & & & \\
\hline Limit & 400 & & & & & & & & \\
\hline K & G & TG & TIG & Mixture & DF up & DF down & $\mathrm{DF}$ av & ROL & Sundt \\
\hline 0 & 18.67 & 23.89 & 24.30 & 22.73 & 20.1635 & 20.6169 & 20.3902 & 20.1351 & 20.2179 \\
\hline 1 & 21.56 & 26.51 & 26.80 & 25.67 & 22.3198 & 22.3680 & 22.3439 & 22.3139 & 22.3302 \\
\hline 2 & 22.24 & 26.81 & 27.12 & 25.92 & 22.4747 & 22.4781 & 22.4764 & 22.4741 & 22.4757 \\
\hline 3 & 22.42 & 26.84 & 27.16 & 25.93 & 22.4830 & 22.4832 & 22.4831 & 22.4830 & 22.4831 \\
\hline 5 & 22.48 & 26.85 & 27.17 & 25.92 & 22.4834 & 22.4834 & 22.4834 & 22.4834 & 22.4834 \\
\hline
\end{tabular}


TABLE 5.6

XL PURE PREMIUMS WITH REINSTATEMENTS UNDER VARIOUS DISTRIBUTION APPROXIMATIONS, $\lambda=10, a=2.5, L=0, c=0$

\begin{tabular}{|c|c|c|c|c|c|c|c|c|c|}
\hline Deductible & 100 & & & & & & & & \\
\hline Limit & 200 & & & & & & & & \\
\hline \multirow[t]{6}{*}{ K } & G & TG & TIG & Mixture & DF up & DF down & DF av & ROL & Sundt \\
\hline & 99.95 & 99.48 & 99.44 & 99.58 & 99.21 & 99.84 & 99.53 & 98.66 & 99.61 \\
\hline & 197.50 & 195.41 & 195.35 & 195.58 & 193.77 & 197.34 & 195.55 & 191.52 & 195.58 \\
\hline & 283.05 & 280.25 & 280.26 & 280.22 & 276.42 & 284.91 & 280.67 & 271.91 & 280.17 \\
\hline & 346.84 & 345.54 & 345.65 & 345.28 & 340.34 & 352.29 & 346.31 & 334.36 & 345.24 \\
\hline & 410.25 & 412.07 & 412.11 & 411.96 & 408.71 & 417.04 & 412.88 & 404.00 & 411.98 \\
\hline Deductible & 200 & & & & & & & & \\
\hline Limit & 300 & & & & & & & & \\
\hline \multirow[t]{6}{*}{ K } & G & TG & TIG & Mixture & DF up & DF down & DF av & ROL & Sundt \\
\hline & 69.05 & 69.22 & 69.59 & 68.18 & 66.34 & 71.32 & 68.83 & 65.84 & 67.22 \\
\hline & 94.64 & 98.32 & 98.63 & 97.47 & 95.47 & 98.52 & 96.99 & 94.98 & 96.24 \\
\hline & 103.27 & 106.95 & 107.13 & 106.47 & 104.66 & 105.69 & 105.17 & 104.42 & 105.00 \\
\hline & 106.08 & 109.02 & 109.19 & 108.56 & 106.88 & 107.15 & 107.02 & 106.81 & 106.98 \\
\hline & 107.27 & 109.54 & 109.73 & 108.99 & 107.39 & 107.40 & 107.40 & 107.39 & 107.39 \\
\hline Deductible & 300 & & & & & & & & \\
\hline Limit & 400 & & & & & & & & \\
\hline \multirow[t]{6}{*}{ K } & G & TG & TIG & Mixture & DF up & DF down & DF av & ROL & Sundt \\
\hline & 35.03 & 39.93 & 40.40 & 38.61 & 36.305 & 37.755 & 37.030 & 36.216 & 36.474 \\
\hline & 42.31 & 47.71 & 47.96 & 47.00 & 43.787 & 44.096 & 43.942 & 43.750 & 43.853 \\
\hline & 44.21 & 48.96 & 49.20 & 48.27 & 44.844 & 44.888 & 44.866 & 44.836 & 44.856 \\
\hline & 44.75 & 49.14 & 49.40 & 48.40 & 44.956 & 44.961 & 44.959 & 44.955 & 44.958 \\
\hline & 44.95 & 49.17 & 49.44 & 48.40 & 44.967 & 44.967 & 44.967 & 44.967 & 44.967 \\
\hline
\end{tabular}


TABLE 5.7

XL PURE PREMIUMS WITH REINSTATEMENTS UNDER VARIOUS DISTRIBUTION APPROXIMATIONS, $\lambda=0.5, \alpha=1.2, L=100, c=0$

\begin{tabular}{|c|c|c|c|c|c|c|c|c|c|}
\hline Deductible & 100 & & & & & & & & \\
\hline Limit & 200 & & & & & & & & \\
\hline \multirow[t]{6}{*}{ K } & G & TG & TIG & Mixture & DF up & DF down & DF av & ROL & Sundt \\
\hline & 4.57 & 4.51 & 4.34 & 4.98 & 4.18 & 3.87 & 4.02 & 4.23 & 4.09 \\
\hline & 5.68 & 5.10 & 4.95 & 5.54 & 4.60 & 4.17 & 4.39 & 4.68 & 4.48 \\
\hline & 5.97 & 5.18 & 5.04 & 5.58 & 4.63 & 4.19 & 4.41 & 4.71 & 4.51 \\
\hline & 6.05 & 5.19 & 5.05 & 5.57 & 4.63 & 4.19 & 4.41 & 4.71 & 4.51 \\
\hline & 6.07 & 5.19 & 5.05 & 5.57 & 4.63 & 4.19 & 4.41 & 4.71 & 4.51 \\
\hline Deductible & 200 & & & & & & & & \\
\hline Limit & 300 & & & & & & & & \\
\hline \multirow[t]{6}{*}{ K } & G & TG & TIG & Mixture & DF up & DF down & DF av & ROL & Sundt \\
\hline & 2.12 & 1.82 & 1.73 & 2.07 & 1.277 & 1.109 & 1.193 & 1.284 & 1.265 \\
\hline & 2.63 & 2.01 & 1.94 & 2.23 & 1.348 & 1.165 & 1.256 & 1.356 & 1.333 \\
\hline & 2.77 & 2.04 & 1.97 & 2.22 & 1.351 & 1.167 & 1.259 & 1.359 & 1.336 \\
\hline & 2.81 & 2.04 & 1.98 & 2.22 & 1.351 & 1.167 & 1.259 & 1.359 & 1.336 \\
\hline & 2.82 & 2.04 & 1.98 & 2.22 & 1.351 & 1.167 & 1.259 & 1.359 & 1.336 \\
\hline Deductible & 300 & & & & & & & & \\
\hline Limit & 400 & & & & & & & & \\
\hline \multirow[t]{6}{*}{ K } & G & TG & TIG & Mixture & DF up & DF down & DF av & ROL & Sundt \\
\hline & 1.34 & 1.06 & 1.01 & 1.22 & 0.583 & 0.523 & 0.553 & 0.584 & 0.579 \\
\hline & 1.67 & 1.17 & 1.12 & 1.29 & 0.604 & 0.542 & 0.573 & 0.606 & 0.601 \\
\hline & 1.75 & 1.18 & 1.14 & 1.28 & 0.605 & 0.542 & 0.573 & 0.607 & 0.601 \\
\hline & 1.78 & 1.18 & 1.14 & 1.28 & 0.605 & 0.542 & 0.573 & 0.607 & 0.601 \\
\hline & 1.79 & 1.18 & 1.14 & 1.28 & 0.605 & 0.542 & 0.573 & 0.607 & 0.601 \\
\hline
\end{tabular}


TABLE 5.8

XL PURE PREMIUMS WITH REINSTATEMENTS UNDER VARIOUS DISTRIBUTION APPROXIMATIONS, $\lambda=1, \alpha=2.5, L=100, c=0$

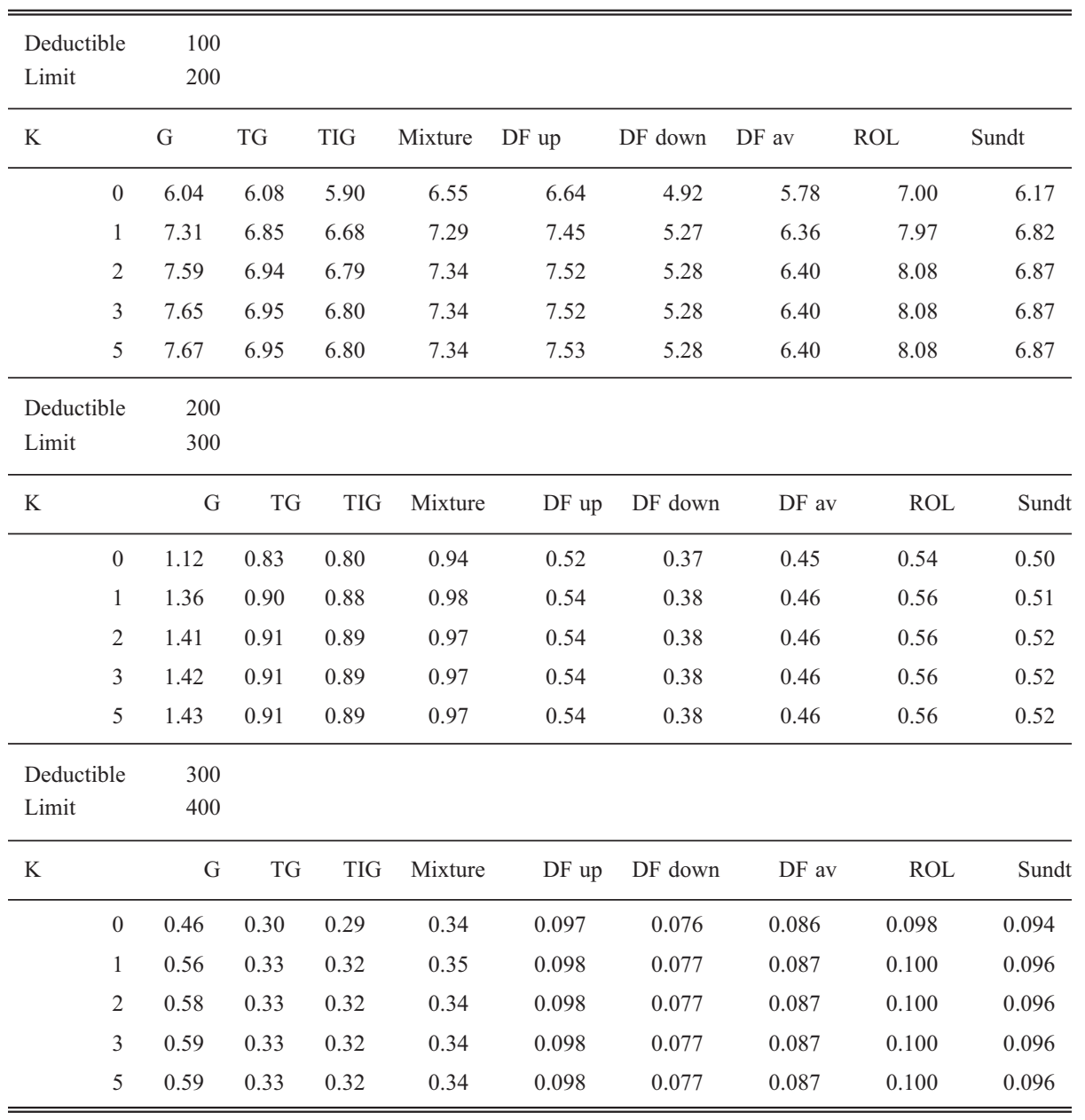


TABLE 5.9

XL PURE PREMIUMS WITH REINSTATEMENTS UNDER VARIOUS DISTRIBUTION APPROXIMATIONS, $\lambda=2, \alpha=2.5, L=100, c=0$

\begin{tabular}{|c|c|c|c|c|c|c|c|c|c|}
\hline Deductible & 100 & & & & & & & & \\
\hline Limit & 200 & & & & & & & & \\
\hline \multirow[t]{6}{*}{ K } & $\mathrm{G}$ & TG & TIG & Mixture & DF up & DF down & DF av & ROL & Sundt \\
\hline & 4.57 & 4.51 & 4.34 & 4.98 & 4.18 & 3.87 & 4.02 & 4.23 & 4.09 \\
\hline & 5.68 & 5.10 & 4.95 & 5.54 & 4.60 & 4.17 & 4.39 & 4.68 & 4.48 \\
\hline & 5.97 & 5.18 & 5.04 & 5.58 & 4.63 & 4.19 & 4.41 & 4.71 & 4.51 \\
\hline & 6.05 & 5.19 & 5.05 & 5.57 & 4.63 & 4.19 & 4.41 & 4.71 & 4.51 \\
\hline & 6.07 & 5.19 & 5.05 & 5.57 & 4.63 & 4.19 & 4.41 & 4.71 & 4.51 \\
\hline Deductible & 200 & & & & & & & & \\
\hline Limit & 300 & & & & & & & & \\
\hline \multirow[t]{6}{*}{ K } & G & TG & TIG & Mixture & DF up & DF down & DF av & ROL & Sundt \\
\hline & 2.12 & 1.82 & 1.73 & 2.07 & 1.277 & 1.109 & 1.193 & 1.284 & 1.265 \\
\hline & 2.63 & 2.01 & 1.94 & 2.23 & 1.348 & 1.165 & 1.256 & 1.356 & 1.333 \\
\hline & 2.77 & 2.04 & 1.97 & 2.22 & 1.351 & 1.167 & 1.259 & 1.359 & 1.336 \\
\hline & 2.81 & 2.04 & 1.98 & 2.22 & 1.351 & 1.167 & 1.259 & 1.359 & 1.336 \\
\hline & 2.82 & 2.04 & 1.98 & 2.22 & 1.351 & 1.167 & 1.259 & 1.359 & 1.336 \\
\hline Deductible & 300 & & & & & & & & \\
\hline Limit & 400 & & & & & & & & \\
\hline \multirow[t]{6}{*}{ K } & G & $\mathrm{TG}$ & TIG & Mixture & DF up & DF down & $\mathrm{DF}$ av & ROL & Sundt \\
\hline & 1.34 & 1.06 & 1.01 & 1.22 & 0.583 & 0.523 & 0.553 & 0.584 & 0.579 \\
\hline & 1.67 & 1.17 & 1.12 & 1.29 & 0.604 & 0.542 & 0.573 & 0.606 & 0.601 \\
\hline & 1.75 & 1.18 & 1.14 & 1.28 & 0.605 & 0.542 & 0.573 & 0.607 & 0.601 \\
\hline & 1.78 & 1.18 & 1.14 & 1.28 & 0.605 & 0.542 & 0.573 & 0.607 & 0.601 \\
\hline & 1.79 & 1.18 & 1.14 & 1.28 & 0.605 & 0.542 & 0.573 & 0.607 & 0.601 \\
\hline
\end{tabular}


TABLE 5.10

XL PURE PREMIUMS WITH REINSTATEMENTS UNDER VARIOUS DISTRIBUTION APPROXIMATIONS, $\lambda=5, \alpha=2.5, L=100, c=0$

\begin{tabular}{|c|c|c|c|c|c|c|c|c|c|}
\hline Deductible & 100 & & & & & & & & \\
\hline Limit & 200 & & & & & & & & \\
\hline \multirow[t]{6}{*}{ K } & G & TG & TIG & Mixture & DF up & DF down & DF av & ROL & Sundt \\
\hline & 65.34 & 66.95 & 67.15 & 66.44 & 65.01 & 69.12 & 67.06 & 63.43 & 66.43 \\
\hline & 98.42 & 102.58 & 102.75 & 102.13 & 101.31 & 104.43 & 102.87 & 99.94 & 102.23 \\
\hline & 112.43 & 117.04 & 117.11 & 116.87 & 117.31 & 117.04 & 117.17 & 117.13 & 116.97 \\
\hline & 117.75 & 121.79 & 121.83 & 121.70 & 123.03 & 120.34 & 121.68 & 123.90 & 121.77 \\
\hline & 120.26 & 123.44 & 123.50 & 123.29 & 125.17 & 121.11 & 123.14 & 126.85 & 123.34 \\
\hline Deductible & 200 & & & & & & & & \\
\hline Limit & 300 & & & & & & & & \\
\hline \multirow[t]{6}{*}{ K } & G & TG & TIG & Mixture & DF up & DF down & $\mathrm{DF}$ av & ROL & Sundt \\
\hline & 9.12 & 9.93 & 9.71 & 10.54 & 10.04 & 8.13 & 9.09 & 10.16 & 9.81 \\
\hline & 11.49 & 11.62 & 11.38 & 12.29 & 11.70 & 9.24 & 10.47 & 11.90 & 11.32 \\
\hline & 12.13 & 11.88 & 11.66 & 12.49 & 11.90 & 9.35 & 10.63 & 12.12 & 11.50 \\
\hline & 12.31 & 11.92 & 11.71 & 12.50 & 11.92 & 9.36 & 10.64 & 12.15 & 11.51 \\
\hline & 12.38 & 11.92 & 11.72 & 12.49 & 11.92 & 9.36 & 10.64 & 12.15 & 11.51 \\
\hline Deductible & 300 & & & & & & & & \\
\hline Limit & 400 & & & & & & & & \\
\hline \multirow[t]{6}{*}{ K } & G & TG & TIG & Mixture & DF up & DF down & DF av & ROL & Sundt \\
\hline & 2.89 & 2.62 & 2.50 & 2.95 & 2.16 & 1.75 & 1.95 & 2.18 & 2.11 \\
\hline & 3.57 & 2.91 & 2.81 & 3.20 & 2.31 & 1.86 & 2.09 & 2.34 & 2.26 \\
\hline & 3.75 & 2.95 & 2.86 & 3.20 & 2.32 & 1.87 & 2.09 & 2.35 & 2.27 \\
\hline & 3.80 & 2.95 & 2.87 & 3.20 & 2.32 & 1.87 & 2.09 & 2.35 & 2.27 \\
\hline & 3.82 & 2.95 & 2.87 & 3.20 & 2.32 & 1.87 & 2.09 & 2.35 & 2.27 \\
\hline
\end{tabular}


TABLE 5.11

XL PURE PREMIUMS WITH REINSTATEMENTS UNDER VARIOUS DISTRIBUTION APPROXIMATIONS, $\lambda=10, a=2.5, L=100, c=0$

\begin{tabular}{|c|c|c|c|c|c|c|c|c|c|}
\hline $\begin{array}{l}\text { Deductible } \\
\text { Limit }\end{array}$ & $\begin{array}{l}100 \\
200\end{array}$ & & & & & & & & \\
\hline \multirow[t]{6}{*}{$\mathrm{K}$} & G & TG & TIG & Mixture & DF up & DF down & $\mathrm{DF}$ av & ROL & Sundt \\
\hline & 97.55 & 95.93 & 95.91 & 96.00 & 94.56 & 97.50 & 96.03 & 92.86 & 95.97 \\
\hline & 183.10 & 180.77 & 180.82 & 180.64 & 177.21 & 185.07 & 181.14 & 173.25 & 180.56 \\
\hline & 246.89 & 246.06 & 246.21 & 245.70 & 241.12 & 252.45 & 246.78 & 235.71 & 245.63 \\
\hline & 287.54 & 288.79 & 288.94 & 288.42 & 284.18 & 295.15 & 289.66 & 278.85 & 288.40 \\
\hline & 321.79 & 324.01 & 324.04 & 323.93 & 322.35 & 326.54 & 324.44 & 319.88 & 323.92 \\
\hline Deductible & 200 & & & & & & & & \\
\hline Limit & 300 & & & & & & & & \\
\hline \multirow[t]{6}{*}{ K } & G & TG & TIG & Mixture & DF up & DF down & DF av & ROL & Sundt \\
\hline & 25.59 & 29.10 & 29.04 & 29.30 & 29.13 & 27.20 & 28.17 & 29.14 & 29.02 \\
\hline & 34.22 & 37.74 & 37.54 & 38.30 & 38.32 & 34.37 & 36.35 & 38.59 & 37.78 \\
\hline & 37.03 & 39.81 & 39.60 & 40.39 & 40.54 & 35.83 & 38.19 & 40.97 & 39.77 \\
\hline & 37.94 & 40.24 & 40.05 & 40.76 & 40.98 & 36.06 & 38.52 & 41.46 & 40.12 \\
\hline & 38.31 & 40.34 & 40.16 & 40.81 & 41.06 & 36.09 & 38.57 & 41.56 & 40.18 \\
\hline Deductible & 300 & & & & & & & & \\
\hline Limit & 400 & & & & & & & & \\
\hline \multirow[t]{6}{*}{ K } & G & TG & TIG & Mixture & DF up & DF down & DF av & ROL & Sundt \\
\hline & 7.27 & 7.78 & 7.56 & 8.39 & 7.48 & 6.34 & 6.91 & 7.53 & 7.38 \\
\hline & 9.18 & 9.02 & 8.80 & 9.65 & 8.54 & 7.13 & 7.84 & 8.62 & 8.38 \\
\hline & 9.71 & 9.21 & 9.01 & 9.78 & 8.65 & 7.21 & 7.93 & 8.74 & 8.48 \\
\hline & 9.87 & 9.24 & 9.04 & 9.79 & 8.66 & 7.21 & 7.94 & 8.75 & 8.49 \\
\hline & 9.93 & 9.24 & 9.05 & 9.78 & 8.66 & 7.21 & 7.94 & 8.75 & 8.49 \\
\hline
\end{tabular}


TABLE 5.12

XL PURE PREMIUMS WITH REINSTATEMENTS UNDER VARIOUS DISTRIBUTION APPROXIMATIONS, $\lambda=0.5, \alpha=1.2, L=200, c=0$

\begin{tabular}{|c|c|c|c|c|c|c|c|c|c|}
\hline Deductible & 100 & & & & & & & & \\
\hline Limit & 200 & & & & & & & & \\
\hline \multirow[t]{6}{*}{ K } & G & TG & TIG & Mixture & DF up & DF down & DF av & ROL & Sundt \\
\hline & 1.10 & 0.59 & 0.60 & 0.5576 & 0.42 & 0.31 & 0.36 & 0.44 & 0.40 \\
\hline & 1.39 & 0.67 & 0.69 & 0.59206 & 0.46 & 0.33 & 0.39 & 0.48 & 0.42 \\
\hline & 1.47 & 0.68 & 0.71 & 0.58855 & 0.46 & 0.33 & 0.39 & 0.48 & 0.43 \\
\hline & 1.49 & 0.68 & 0.71 & 0.58662 & 0.46 & 0.33 & 0.39 & 0.48 & 0.43 \\
\hline & 1.50 & 0.68 & 0.71 & 0.586 & 0.46 & 0.33 & 0.39 & 0.48 & 0.43 \\
\hline Deductible & 200 & & & & & & & & \\
\hline Limit & 300 & & & & & & & & \\
\hline \multirow[t]{6}{*}{ K } & G & TG & TIG & Mixture & DF up & DF down & DF av & ROL & Sundt \\
\hline & 0.51 & 0.19667 & 0.21 & 0.15 & 0.07 & 0.05586 & 0.06310 & 0.07154 & 0.06822 \\
\hline & 0.64 & 0.2189 & 0.24 & 0.15 & 0.07 & 0.05796 & 0.06560 & 0.07455 & 0.07096 \\
\hline & 0.68 & 0.22147 & 0.25 & 0.15 & 0.07 & 0.05803 & 0.06568 & 0.07465 & 0.07105 \\
\hline & 0.69 & 0.22177 & 0.25 & 0.15 & 0.07 & 0.05803 & 0.06569 & 0.07465 & 0.07105 \\
\hline & 0.70 & 0.22181 & 0.25 & 0.15 & 0.07 & 0.05803 & 0.06569 & 0.07465 & 0.07105 \\
\hline Deductible & 300 & & & & & & & & \\
\hline Limit & 400 & & & & & & & & \\
\hline \multirow[t]{6}{*}{ K } & G & $\mathrm{TG}$ & TIG & Mixture & DF up & DF down & DF av & ROL & Sundt \\
\hline & 0.32 & 0.11 & 0.12 & 0.07 & 0.02146 & 0.01816 & 0.01981 & 0.02165 & 0.02110 \\
\hline & 0.41 & 0.12 & 0.13 & 0.06 & 0.02205 & 0.01863 & 0.02034 & 0.02225 & 0.02168 \\
\hline & 0.43 & 0.12 & 0.14 & 0.06 & 0.02207 & 0.01864 & 0.02036 & 0.02227 & 0.02169 \\
\hline & 0.44 & 0.12 & 0.14 & 0.06 & 0.02207 & 0.01864 & 0.02036 & 0.02227 & 0.02169 \\
\hline & 0.44 & 0.12 & 0.14 & 0.06 & 0.02207 & 0.01864 & 0.02036 & 0.02227 & 0.02169 \\
\hline
\end{tabular}


TABLE 5.13

XL PURE PREMIUMS WITH REINSTATEMENTS UNDER VARIOUS DISTRIBUTION APPROXIMATIONS, $\lambda=1, \alpha=2.5, L=200, c=0$

\begin{tabular}{|c|c|c|c|c|c|c|c|c|c|}
\hline Deductible & 100 & & & & & & & & \\
\hline Limit & 200 & & & & & & & & \\
\hline \multirow[t]{6}{*}{ K } & G & TG & TIG & Mixture & DF up & DF down & DF av & ROL & Sundt \\
\hline & 1.26517 & 0.77162 & 0.78 & 0.74455 & 0.80789 & 0.34866 & 0.57828 & 0.96905 & 0.65004 \\
\hline & 1.54479 & 0.86172 & 0.89 & 0.79584 & 0.87970 & 0.36127 & 0.62049 & 1.07113 & 0.69867 \\
\hline & 1.60832 & 0.87178 & 0.90 & 0.79438 & 0.88469 & 0.36162 & 0.62316 & 1.07980 & 0.70147 \\
\hline & 1.623 & 0.87287 & 0.90 & 0.79298 & 0.88498 & 0.36162 & 0.62330 & 1.08041 & 0.70160 \\
\hline & 1.62725 & 0.87299 & 0.90 & 0.79256 & 0.88499 & 0.36163 & 0.62331 & 1.08045 & 0.70160 \\
\hline Deductible & 200 & & & & & & & & \\
\hline Limit & 300 & & & & & & & & \\
\hline \multirow[t]{6}{*}{ K } & G & $\mathrm{TG}$ & TIG & Mixture & DF up & DF down & DF av & ROL & Sundt \\
\hline & 0.23258 & 0.07 & 0.08 & 0.04008 & 0.01772 & 0.01007 & 0.0139 & 0.01905 & 0.01555 \\
\hline & 0.28685 & 0.08 & 0.09 & 0.03636 & 0.01817 & 0.01026 & 0.01422 & 0.01956 & 0.01590 \\
\hline & 0.3003 & 0.08 & 0.10 & 0.03478 & 0.01818 & 0.01026 & 0.01422 & 0.01957 & 0.01590 \\
\hline & 0.30375 & 0.08 & 0.10 & 0.03443 & 0.01818 & 0.01026 & 0.01422 & 0.01957 & 0.01590 \\
\hline & 0.30491 & 0.08 & 0.10 & 0.03435 & 0.01818 & 0.01026 & 0.01422 & 0.01957 & 0.01590 \\
\hline Deductible & 300 & & & & & & & & \\
\hline Limit & 400 & & & & & & & & \\
\hline \multirow[t]{6}{*}{ K } & G & TG & TIG & Mixture & DF up & DF down & DF av & ROL & Sundt \\
\hline & 0.10 & 0.02 & 0.03 & 0.01 & 0.00141 & 0.00095 & 0.00118 & 0.00147 & 0.00131 \\
\hline & 0.12 & 0.03 & 0.03 & 0.01 & 0.00142 & 0.00096 & 0.00119 & 0.00148 & 0.00132 \\
\hline & 0.13 & 0.03 & 0.03 & 0.01 & 0.00142 & 0.00096 & 0.00119 & 0.00148 & 0.00132 \\
\hline & 0.13 & 0.03 & 0.03 & 0.01 & 0.00142 & 0.00096 & 0.00119 & 0.00148 & 0.00132 \\
\hline & 0.13 & 0.03 & 0.03 & 0.01 & 0.00142 & 0.00096 & 0.00119 & 0.00148 & 0.00132 \\
\hline
\end{tabular}


TABLE 5.14

XL PURE PREMIUMS WITH REINSTATEMENTS UNDER VARIOUS DISTRIBUTION APPROXIMATIONS, $\lambda=2, a=2.5, L=200, c=0$

\begin{tabular}{|c|c|c|c|c|c|c|c|c|c|}
\hline Deductible & 100 & & & & & & & & \\
\hline Limit & 200 & & & & & & & & \\
\hline \multirow[t]{6}{*}{ K } & G & TG & TIG & Mixture & DF up & DF down & DF av & ROL & Sundt \\
\hline & 4.85 & 4.33077 & 4.26 & 4.52 & 5.06 & 3.16 & 4.11 & 5.67 & 4.41 \\
\hline & 6.12 & 5.09722 & 5.04 & 5.24 & 5.97 & 3.49 & 4.73 & 6.84 & 5.09 \\
\hline & 6.46 & 5.21701 & 5.18 & 5.32 & 6.09 & 3.51 & 4.80 & 7.03 & 5.18 \\
\hline & 6.54 & 5.23424 & 5.20 & 5.33 & 6.11 & 3.51 & 4.81 & 7.06 & 5.18 \\
\hline & 6.57 & 5.23687 & 5.20 & 5.32 & 6.11 & 3.51 & 4.81 & 7.07 & 5.18 \\
\hline Deductible & 200 & & & & & & & & \\
\hline Limit & 300 & & & & & & & & \\
\hline \multirow[t]{6}{*}{ K } & G & TG & TIG & Mixture & DF up & DF down & DF av & ROL & Sundt \\
\hline & 0.57 & 0.24 & 0.26 & 0.19 & 0.13 & 0.08 & 0.11 & 0.14 & 0.12 \\
\hline & 0.71 & 0.27 & 0.29 & 0.20 & 0.14 & 0.08 & 0.11 & 0.15 & 0.12 \\
\hline & 0.74 & 0.27 & 0.30 & 0.19 & 0.14 & 0.08 & 0.11 & 0.15 & 0.12 \\
\hline & 0.75 & 0.27 & 0.30 & 0.19 & 0.14 & 0.08 & 0.11 & 0.15 & 0.12 \\
\hline & 0.76 & 0.27 & 0.30 & 0.19 & 0.14 & 0.08 & 0.11 & 0.15 & 0.12 \\
\hline Deductible & 300 & & & & & & & & \\
\hline Limit & 400 & & & & & & & & \\
\hline \multirow[t]{6}{*}{ K } & G & TG & TIG & Mixture & DF up & DF down & DF av & ROL & Sundt \\
\hline & 0.21 & 0.06 & 0.07 & 0.03 & 0.0109 & 0.0075 & 0.0092 & 0.0113 & 0.0102 \\
\hline & 0.27 & 0.07 & 0.08 & 0.03 & 0.0112 & 0.0076 & 0.0094 & 0.0116 & 0.0104 \\
\hline & 0.28 & 0.07 & 0.08 & 0.03 & 0.0112 & 0.0076 & 0.0094 & 0.0116 & 0.0104 \\
\hline & 0.29 & 0.07 & 0.08 & 0.03 & 0.0112 & 0.0076 & 0.0094 & 0.0116 & 0.0104 \\
\hline & 0.29 & 0.07 & 0.09 & 0.03 & 0.0112 & 0.0076 & 0.0094 & 0.0116 & 0.0104 \\
\hline
\end{tabular}


TABLE 5.15

XL PURE PREMIUMS WITH REINSTATEMENTS UNDER VARIOUS DISTRIBUTION APPROXIMATIONS, $\lambda=5, \alpha=2.5, L=200, c=0$

\begin{tabular}{|c|c|c|c|c|c|c|c|c|c|}
\hline Deductible & 100 & & & & & & & & \\
\hline Limit & 200 & & & & & & & & \\
\hline \multirow[t]{6}{*}{ K } & G & TG & TIG & Mixture & DF up & DF down & DF av & ROL & Sundt \\
\hline & 33.08 & 35.6228 & 35.60 & 35.69 & 36.30 & 35.31 & 35.81 & 36.51 & 35.80 \\
\hline & 47.09 & 50.087 & 49.96 & 50.42 & 52.30 & 47.92 & 50.11 & 53.70 & 50.54 \\
\hline & 52.41 & 54.8393 & 54.67 & 55.26 & 58.02 & 51.22 & 54.62 & 60.47 & 55.35 \\
\hline & 54.29 & 56.1651 & 56.01 & 56.56 & 59.72 & 51.88 & 55.80 & 62.75 & 56.63 \\
\hline & 55.13 & 56.5627 & 56.43 & 56.90 & 60.25 & 52.00 & 56.13 & 63.60 & 56.97 \\
\hline \multirow{2}{*}{$\begin{array}{l}\text { Deductible } \\
\text { Limit }\end{array}$} & 200 & & & & & & & & \\
\hline & 300 & & & & & & & & \\
\hline K & G & TG & TIG & Mixture & DF up & DF down & DF av & ROL & Sundt \\
\hline 0 & 2.37 & 1.69 & 1.68 & 1.74142 & 1.65 & 1.11 & 1.38 & 1.74 & 1.51 \\
\hline 1 & 3.01 & 1.95 & 1.96 & 1.94238 & 1.86 & 1.22 & 1.54 & 1.96 & 1.69 \\
\hline 2 & 3.19 & 1.99 & 2.00 & 1.95325 & 1.88 & 1.23 & 1.55 & 1.99 & 1.70 \\
\hline 3 & 3.24 & 1.99 & 2.01 & 1.95088 & 1.88 & 1.23 & 1.55 & 1.99 & 1.70 \\
\hline 5 & 3.26 & 2.00 & 2.01 & 1.94951 & 1.88 & 1.23 & 1.55 & 1.99 & 1.70 \\
\hline \multirow{2}{*}{$\begin{array}{l}\text { Deductible } \\
\text { Limit }\end{array}$} & 300 & & & & & & & & \\
\hline & 400 & & & & & & & & \\
\hline \multirow[t]{6}{*}{ K } & G & TG & TIG & Mixture & DF up & DF down & DF av & ROL & Sundt \\
\hline & 0.68 & 0.30 & 0.31 & 0.25 & 0.155 & 0.110 & 0.132 & 0.160 & 0.145 \\
\hline & 0.86 & 0.33 & 0.36 & 0.26 & 0.163 & 0.115 & 0.139 & 0.169 & 0.153 \\
\hline & 0.90 & 0.34 & 0.37 & 0.25 & 0.164 & 0.115 & 0.139 & 0.169 & 0.153 \\
\hline & 0.92 & 0.34 & 0.37 & 0.25 & 0.164 & 0.115 & 0.139 & 0.170 & 0.153 \\
\hline & 0.92 & 0.34 & 0.37 & 0.25 & 0.164 & 0.115 & 0.139 & 0.170 & 0.153 \\
\hline
\end{tabular}


TABLE 5.16

XL PURE PREMIUMS WITH REINSTATEMENTS UNDER VARIOUS DISTRIBUTION APPROXIMATIONS,

$$
\lambda=10, \alpha=2.5, L=200, c=0
$$

\begin{tabular}{|c|c|c|c|c|c|c|c|c|c|}
\hline Deductible & 100 & & & & & & & & \\
\hline Limit & 200 & & & & & & & & \\
\hline \multirow[t]{6}{*}{ K } & G & TG & TIG & Mixture & DF up & DF down & $\mathrm{DF}$ av & ROL & Sundt \\
\hline & 85.56 & 84.8358 & 84.91 & 84.65 & 82.64 & 87.58 & 85.11 & 80.39 & 84.59 \\
\hline & 149.34 & 150.133 & 150.30 & 149.71 & 146.56 & 154.95 & 150.75 & 142.84 & 149.66 \\
\hline & 190.00 & 192.859 & 193.03 & 192.43 & 189.61 & 197.65 & 193.63 & 185.99 & 192.43 \\
\hline & 212.76 & 216.654 & 216.76 & 216.38 & 214.83 & 219.71 & 217.27 & 212.48 & 216.41 \\
\hline & 229.59 & 232.892 & 232.93 & 232.79 & 233.60 & 232.33 & 232.97 & 234.19 & 232.79 \\
\hline Deductible & 200 & & & & & & & & \\
\hline Limit & 300 & & & & & & & & \\
\hline \multirow[t]{6}{*}{ K } & G & TG & TIG & Mixture & DF up & DF down & DF av & ROL & Sundt \\
\hline & 8.63 & 8.63 & 8.50 & 8.99961 & 9.19 & 7.17 & 8.18 & 9.44 & 8.76 \\
\hline & 11.45 & 10.70 & 10.57 & 11.0888 & 11.42 & 8.63 & 10.02 & 11.83 & 10.74 \\
\hline & 12.35 & 11.14 & 11.02 & 11.4655 & 11.85 & 8.85 & 10.35 & 12.32 & 11.10 \\
\hline & 12.63 & 11.22 & 11.11 & 11.5156 & 11.92 & 8.88 & 10.40 & 12.41 & 11.15 \\
\hline & 12.75 & 11.23 & 11.13 & 11.5177 & 11.93 & 8.88 & 10.41 & 12.42 & 11.16 \\
\hline Deductible & 300 & & & & & & & & \\
\hline Limit & 400 & & & & & & & & \\
\hline \multirow[t]{6}{*}{ K } & G & $\mathrm{TG}$ & TIG & Mixture & DF up & DF down & $\mathrm{DF}$ av & ROL & Sundt \\
\hline & 1.91 & 1.25 & 1.24 & 1.26 & 1.056 & 0.792 & 0.924 & 1.086 & 1.004 \\
\hline & 2.44 & 1.43 & 1.45 & 1.39 & 1.169 & 0.865 & 1.017 & 1.205 & 1.105 \\
\hline & 2.60 & 1.46 & 1.48 & 1.40 & 1.179 & 0.870 & 1.024 & 1.216 & 1.114 \\
\hline & 2.64 & 1.46 & 1.49 & 1.39 & 1.179 & 0.870 & 1.025 & 1.216 & 1.114 \\
\hline & 2.66 & 1.46 & 1.49 & 1.39 & 1.179 & 0.870 & 1.025 & 1.216 & 1.114 \\
\hline
\end{tabular}

\section{REFERENCES}

ChaubeY, Y.P., (1989) Edgeworth expansions with mixtures and applications. Metron, XLVII, 5364.

Chaubey Y.P., GarRido, J. and Trudeau, S. (1998) On the computation of aggregate claims distributions: some new approximations. Insurance: Mathematics and Economics, 23, 215-230.

DicKSON, D.C.M. and WATERS, H.R. (1993) Gamma processes and finite time survival probabilities. ASTIN Bulletin, 23(2), 259-272.

Hess, K.Th. and Schmidt, K.D. (2004) Optimal premium plans for reinsurance with reinstatements. ASTIN Bulletin, 34(2), 299-313.

HÜrLIMANN, W. (1996) Improved analytical bounds for some risk quantities. ASTIN Bulletin, 26(2), $185-99$. 
HürLIMANN, W. (2001) Truncated linear zero utility pricing and actuarial protection models. Blätter der Deutschen Gesellschaft für Versicherungsmathematik, XXV, 271-279.

HürLimanN, W. (2003) Conditional value-at-risk bounds for compound Poisson risks and a normal approximation. Journal of Applied Mathematics, 3(3), 141-154.

MatA, A.J. (2000) Pricing Excess of Loss Reinsurance with Reinstatements. ASTIN Bulletin, 30(2), 349-368.

SimON, L.J. (1972) Actuarial applications in catastrophe reinsurance. Proceedings of the Casualty Actuarial Society LIX, 196-202. Discussions. Proceedings of the Casualty Actuarial Society, LX, 137-156.

SundT, B. (1991) On excess of loss reinsurance with reinstatements. Bulletin of the Swiss Association of Actuaries, Heft 1, 51-65.

WalHin, J.-F. (2001) Some comments on two approximations used for the pricing of reinstatements. Bulletin of the Swiss Association of Actuaries, Heft 1, 29-47.

WALHIN, J.-F. (2002) On the use of the multivariate stochastic order in risk theory. Blätter der Deutschen Gesellschaft für Versicherungsmathematik, XXV, 503-519.

Walhin, J.-F. and PARIS, J. (2000) The effect of excess-of-loss reinsurance with reinstatements on the cedant's portfolio. Blätter der Deutschen Gesellschaft für Versicherungsmathematik, XXIV, 615-627.

WALHIN, J.-F. and PARIS, J. (2001a) Excess of loss reinsurance with reinstatements: premium calculation and ruin probability of the cedant. Blätter der Deutschen Gesellschaft für Versicherungsmathematik, XXV, 1-12.

Walhin, J.-F. and PARIS, J. (2001b). Some comments on the individual risk model and multivariate extension. Blätter der Deutschen Gesellschaft für Versicherungsmathematik, XXV, 257-270.

WERNER HÜRLIMANN

Feldstrasse 145

CH-8004 Zürich

Switzerland

Tel.: +416120606 64

E-mail:werner.huerlimann@aon.ch

URL: www.geocities.com/hurlimann53 